LAWRENCE LIVERMORE NATIONAL LABORATORY

Self-Consistent Simulation of Turbulence and Transport in Tokamak Edge Plasmas

T. D. Rognlien, M. V. Umansky, X. Q. Xu, and R. H. Cohen

September 3, 2003

Plasma Edge Theory Conference, San Diego, California, September 3-5, 2003 


\section{DISCLAIMER}

This document was prepared as an account of work sponsored by an agency of the United States Government. Neither the United States Government nor the University of California nor any of their employees, makes any warranty, express or implied, or assumes any legal liability or responsibility for the accuracy, completeness, or usefulness of any information, apparatus, product, or process disclosed, or represents that its use would not infringe privately owned rights. Reference herein to any specific commercial product, process, or service by trade name, trademark, manufacturer, or otherwise, does not necessarily constitute or imply its endorsement, recommendation, or favoring by the United States Government or the University of California. The views and opinions of authors expressed herein do not necessarily state or reflect those of the United States Government or the University of California, and shall not be used for advertising or product endorsement purposes.

This is a preprint of a paper intended for publication in a journal or proceedings. Since changes may be made before publication, this preprint is made available with the understanding that it will not be cited or reproduced without the permission of the author.

This report has been reproduced directly from the best available copy.

Available electronically at http://www.doc.gov/bridge

Available for a processing fee to U.S. Department of Energy

And its contractors in paper from

U.S. Department of Energy

Office of Scientific and Technical Information

P.O. Box 62

Oak Ridge, TN 37831-0062

Telephone: (865) 576-8401

Facsimile: (865) 576-5728

E-mail: reports@adonis.osti.gov

Available for the sale to the public from

U.S. Department of Commerce

National Technical Information Service

5285 Port Royal Road

Springfield, VA 22161

Telephone: (800) 553-6847

Facsimile: (703) 605-6900

E-mail: orders@ntis.fedworld.gov

Online ordering: http:/ / www.ntis.gov/ordering.htm

\section{OR}

Lawrence Livermore National Laboratory

Technical Information Department's Digital Library

http: / / www.llnl.gov/tid/Library.html 


\title{
Self-consistent simulation of turbulence and transport in toka- mak edge plasmas
}

\author{
T.D. Rognlien*, M.V. Umansky, X.Q. Xu, and R.H. Cohen \\ University of California Lawrence Livermore National Laboratory, Livermore, CA 94551 USA
}

Received 3 September, 2003

Key words tokamak edge-plasma, fluid-plasma turbulence, edge-plasma transport

PACS 53.35Ra, 52.65.Kj, 52.40.Hf

The status of coupling the fluid 3D turbulence code BOUT and the fluid plasma/neutral 2D transport code UEDGE is reported, where both codes simulate the edge region of diverted tokamaks from several $\mathrm{cm}$ inside the magnetic separatrix to the far scrape-off layer (SOL), thereby including the magnetic X-point. Because the characteristic time scale of the turbulence is short $\left(\sim 10^{-5}-10^{-4} \mathrm{~s}\right)$ and the profile evolution time scale can be long $\left(\sim 10^{-2}-10^{-1} \mathrm{~s}\right.$ owing to recycling), an iterative scheme is used that relaxes the turbulent fluxes passed from BOUT to UEDGE and the profiles from UEDGE to BOUT over many coupling steps. Each code is run on its own characteristic time scale, yielding a statistically averaged steady state. For this initial study, the ion and neutral densities and parallel velocities are evolved, while the temperature profiles are stationary. Here the turbulence code is run in the electrostatic approximation. For this example of self-consistent coupling with strong L-mode-like turbulence, the ion flux to the main-chamber exceeds that to the divertor plates.

\section{Introduction}

In tokamak edge plasmas, the profiles of mean particle density, momentum, and energy are often strongly influenced by plasma turbulence that produces enhanced radial transport. In turn, the turbulence is driven by the plasma gradients that are partially determined by the enhanced transport. Suppression of the turbulence for large injected core power gives rise to an edge transport barrier with improved core confinement, the so-called H-mode. A predictive model that describes the profile/turbulence interaction, and the conditions that give rise to the transition from the more turbulent L-mode to the H-mode, requires self-consistent coupling of the turbulence and transport models.

A substantial time-scale separation can exist between the characteristic time of the turbulence and the plasma profile adjustment that includes recycling of ions into neutral gas at surfaces. Thus, we illustrate a method of coupling the 3D fluid turbulence code BOUT [1] and the 2D transport code UEDGE [2] where each system is evolved on it own characteristic time scale via an iterative coupling that utilizes a relaxation procedure [3]. The coupling can yield a statistical steady-state in an efficient manner since the long time-scale transport code runs very fast compared to the short time-scale turbulence code. The common UEDGE/BOUT mesh in the poloidal plane is shown in Fig. 1, which facilitates the coupling.

The calculations presented provide a first step in developing a self-consistent edge-plasma simulation model through coupling the radial ion particle flux and the resulting long-time density profile. Since the neutral particles can play an important role in the profile evolution, we utilize the fluid neutral model within UEDGE to maintain consistency of the neutral density spatial variation.

The paper presents the simulation model in Sec. 2 and sample results in Sec. 3. A discussion of the implications and future directions of this work, along with a summary, is provided in Sec. 4.

* Corresponding author: e-mail: trognlien@IInl.gov, Phone: 925-422-9830 Fax: 925-423-3484 


\section{Equations and coupling algorithm}

\subsection{Transport equations}

The transport code evolves the toroidally symmetric component of the ion and neutral densities $\left(N_{i}\right.$ and $\left.N_{g}\right)$ and the associated parallel velocities $\left(V_{\| i}\right.$ and $\left.V_{\| g}\right)$. The ion and neutral density $\left(N_{i}\right)$ continuity equations are

$$
\frac{\partial N_{k}}{\partial t}+\nabla \cdot\left(N_{k} \mathbf{V}_{k}\right)=S_{p k}
$$

where $k=i$ for ions and $k=g$ for neutrals. The source/sink terms from ionization and recombination for ions and neutrals just balance, i.e., $S_{p i}=-S_{p g}$. The plasma quasineutrality demands very nearly equal ion and electron densities, $N_{i}=N_{e}$, which is valid for low-frequency turbulence and transport time-scales; thus, we use $N_{i}$ and $N_{e}$ interchangeably for cases with hydrogen ions only. The $S_{p k}$ source terms (and thus neutrals) are only explicitly included in the transport code because the associated collision time in the main chamber is in the range of $10^{-4}-10^{-3} \mathrm{~s}$ for this simulation, which is much longer that the growth rate of the turbulence $\left(\sim 10^{-5} \mathrm{~s}\right)$.

The ion and neutral parallel momentum density $\left(N_{k} m_{k} V_{\| k}\right)$ equations are,

$$
\frac{\partial N_{k} m_{k} V_{\| k}}{\partial t}+\nabla \cdot\left(N_{k} m_{k} V_{\| k} \mathbf{V}_{k}\right)=-\nabla_{\|} P_{k}+q_{k} N_{k} E_{\|}-F_{\| k}-R_{\| k}
$$

Here $m_{k}$ is the mass, $P_{k}=N_{k} T_{k}$ is the pressure, $q_{k}=e$ is the charge for a deuterium ion. For the ions $(k=i), E_{\|} \approx-\nabla_{\|} P_{e} /\left(e N_{i}\right)$ is the electric field, and $R_{i \|}$ is the friction force [4]. For the neutrals, we take $q_{g}=R_{\| g}=0, F_{\| g}=m_{g} N_{g} \nu_{c x}\left(V_{\| g}-V_{\| i}\right)$; an equal, but opposite term appears in the component $F_{\| i}$, in addition to the ion-electron contribution, i.e., $F_{\| i}=-F_{\| g}+(\nabla \cdot \Pi)_{\|}$, where $\Pi$ is the ion stress tensor [4].

The cross-field radial ion velocity comes from BOUT turbulent fluxes by calculating an effective diffusion coefficient, $D_{i}$, via the relation

$$
D_{i}=\max \left[-\Gamma_{r} /\left(d N_{i} / d r\right), 0.01\right]\left(\mathrm{m}^{2} / \mathrm{s}\right),
$$

where $\Gamma_{r}$ is the toroidal and time average of the BOUT particle flux, and $r$ is the radial distance normal to a flux surface. UEDGE then uses the consistent radial velocity $V_{r i}=-\left(D_{i} / N_{i}\right) d N_{i} / d r$. The max function removes any regions of negative, $D_{i}$, which occurs occasionally. Negative $D_{i}$ can be resolved by using a convection term, i.e., $\Gamma_{r}=V_{r c} N_{i}$ to represent at least a portion of the flux. However, for this initial study, we have found the diffusion formulation to be most robust.

For the neutrals, the cross-field velocities come from the inertialess momentum equation including chargeexchange and ionization, yielding

$$
\mathbf{V}_{\perp g}=-\left[T_{g} /\left(m_{g} N_{g} \nu_{g}\right)\right] \nabla_{\perp} N_{g}
$$

where $T_{g}$ is the neutral temperature (usually set to $T_{i}$ ), and $\nu_{g}$ is the sum of the charge-exchange and ionization collision frequencies. In order to prevent unphysically large velocities in low collisionality regions, $\left|V_{r g}\right|$ is flux limited such that it cannot exceed the neutral thermal velocity, $\left(T_{g} / m_{g}\right)^{1 / 2}$.

The ion and electron temperature profiles are fixed and set equal throughout the iteration, although UEDGE can evolve these, which will be done in the future. The profiles are typical of those found for full transport modeling with fixed transport coefficients. In the radial direction, $T_{e, i}$ fall from $180 \mathrm{eV}$ at core boundary to $50 \mathrm{eV}$ at the separatrix, and decrease further to $12 \mathrm{eV}$ at the outer wall. In the poloidal direction, just outside the separatrix, $T_{e, i}$ fall from a maximum of $50 \mathrm{eV}$ at the outer midplane to $\sim 15 \mathrm{eV}$ at the divertor plates. At the plates, $T_{e, i}$ decrease away from the separatrix to $4 \mathrm{eV}$ at the private-flux and outer walls.

\subsection{Turbulence equations}

It is well known that the edge-plasma profiles given rise to various low-frequency instabilities [5], and that the resulting turbulence drives cross-field transport well in excess of that collisional transport. Here the turbulence is simulated by the 3D BOUT code [1], which utilizes a fluid description based on the Braginskii equations [4]. The details of the BOUT turbulence equations are given in Ref. [1]; here we only enumerate the equations solved. The 
ion continuity and parallel momentum equations described in Sec. 2.1 are used without the neutral source terms; lower-case symbols are used to denote fluctuating quantities, e.g., for the density, $N_{i} \rightarrow n_{i}$. In addition, there are equations for the electron parallel momentum with inertia, the plasma vorticity (via the current continuity equation), and separate ion and electron temperature equations. For the electrostatic limit used in this paper, there are six fluctuating field quantities: $n_{i}, v_{\| i}, v_{\| e}, \phi, t_{e}$, and $t_{i}$, where $\phi$ is the electrostatic potential. The electromagnetic version of BOUT [1] includes an additional equation for the parallel magnetic vector potential.

\subsection{Coupling algorithm and time scales}

The coupling between the transport and turbulence is accomplished through an iterative scheme of the type described in Ref. [3]. The total density $\left(=N_{i}+n_{i}\right)$ is the sum of the slowly evolving, toroidally averaged density and the faster fluctuation density. The characteristic time for the slow toroidal average to change is called $\tau_{0}$, while the corresponding time for the turbulence is $\tau_{1}\left(\tau_{1} \ll \tau_{0}\right)$. For the slow transport density, we solve

$$
\frac{\partial N_{i}^{m}}{\partial t}+\nabla \cdot\left(N_{i}^{m} \mathbf{V}_{\| i}^{m}+\boldsymbol{\Gamma}_{\mathbf{r}}^{m-1}\right)=K_{i} N_{e}^{m} N_{g}^{m}-K_{r} N_{i}^{m} N_{e}^{m}
$$

where $m$ is the iteration index, and $K_{i, r}\left(T_{e}, N_{e}\right)$ are the hydrogen ionization and recombination rate coefficients, respectively. The radial plasma flux across flux surfaces is obtained from the turbulence simulation as

$$
\Gamma_{r}^{m-1}=\left(1-\alpha_{1}\right) \Gamma_{r}^{m-2}+\alpha_{1}\left\langle n_{i} v_{r i}\right\rangle^{m-1},
$$

where $\alpha_{1}$ is a relaxation parameter in the range [0,1], and the angled brackets denote a double average over the toroidal direction and time for a period $\tau_{1}$.

The density profile used in the turbulence code to generate the flux $\Gamma_{r}^{m-1}$ is likewise a relaxed combination of previous profiles, i.e.,

$$
N_{i}^{m-1}=\left(1-\alpha_{0}\right) N_{i}^{m-2}+\alpha_{0} N_{i}^{m-1} .
$$

Here $\alpha_{0}$ is a second relaxation parameter. We also pass the turbulence code a relaxed parallel velocity, $V_{\| i}^{m-1}$. The iteration loop is completed by the using UEDGE to obtain $N_{i}^{m}$ from Eq. (5).

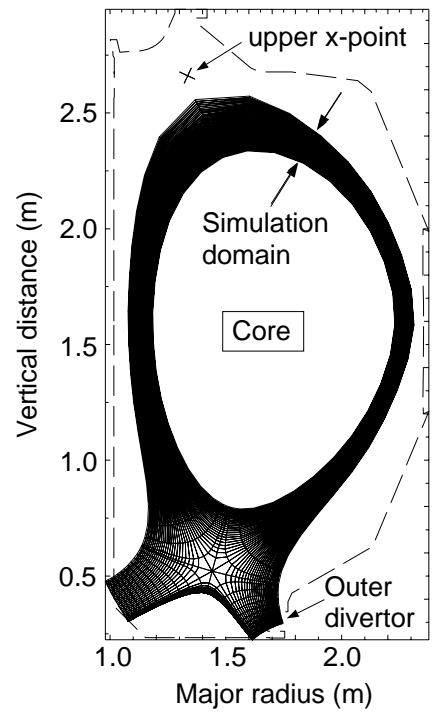

Fig. 1 The common UEDGE/BOUT mesh in the poloidal plane obtained from an MHD equilibrium for DIII-D tokamak discharge 107404.

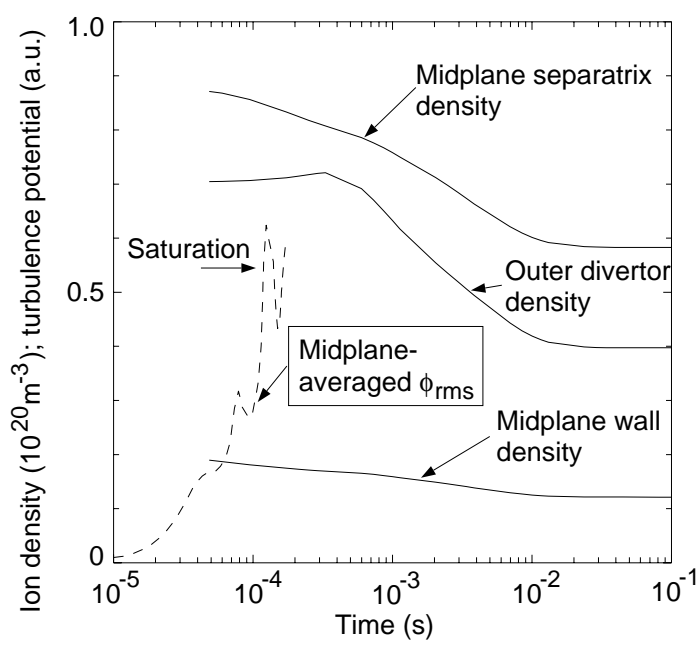

Fig. 2 Comparison of turbulence time scales from the potential fluctuation obtained from BOUT (dashed line) and the toroidally averaged $N_{i}$ densities obtained from UEDGE in changing the turbulent flux $\Gamma_{r}^{m}$ from that found for $m=3$ to that at $m=8$. 
It is typical for the transport time, $\tau_{0}$, to be much longer that the turbulence time, $\tau_{1}$. Thus, it is possible to obtain substantial savings in simulation time to find a self-consistent steady-state by running each system (transport and turbulence) on their own characteristic times. For the system analyzed here, we show the characteristic times from actual simulation results in Fig. 2. The dotted line shows the initial growth and saturation of the fluctuating potential averaged radially and toroidally at the outer midplane, yielding an estimate of $\tau_{1} \sim 2 \times 10^{-5} \mathrm{~s}$ using the e-folding time of the initial fluctuation level. However, a somewhat longer time is obtained if one uses the oscillations in the saturated spectrum observed at the end of the dotted curve, yielding $\tau_{1} \sim 5 \times 10^{-5} \mathrm{~s}$. The solid curves in Fig. 2 show the change in the transport density, $N_{i}$, at several locations by solving only the transport equations starting from the $N_{i}^{m=3}$ profile, but using the later flux $\Gamma_{r}^{m=8}$ (to clearly show substantial evolution). Steady-state for the transport profiles indicates that $\tau_{0} \sim 10^{-2} \mathrm{~s}$. In the simulations to follow, we allow $\tau_{0} \rightarrow 1 \mathrm{~s}$, since there is little added computation cost in extending this time range with the implicit time-integrator in UEDGE.

\section{Simulations results}

As an example of the coupling procedure, we consider the configuration from the DIII-D tokamak for discharge 107404 shown in Fig. 1. The interior boundary has a poloidal magnetic flux of 0.9 normalized to that on the separatrix, and the outer wall has a flux value of 1.1 . The core-edge deuterium density is set to $2.5 \times 10^{19} \mathrm{~m}^{-3}$, and the corresponding $T_{e, i}=180 \mathrm{eV}$. The particle recycling coefficient on the divertor plates is $R_{p}=0.98$, while on the outer wall, $R_{w}=0.90$; the lower value on the wall is to indicate that the wall may do some pumping and regions of it are quite remote from the outer flux surface in the simulation domain. The ion density is given a $2 \mathrm{~cm}$ radial scale-length at the private-flux and outer walls.

In addition to the effective diffusion coefficient obtained from the turbulent flux, $\Gamma_{r}$, in Eq. (3), we add a small auxiliary diffusion coefficient to the core region that decreases linearly from $0.2 \mathrm{~m}^{2} / \mathrm{s}$ at the core boundary to zero at the separatrix. This auxiliary term was added to provide a reasonable density profile near the core boundary during the initial stages of the iterations; it may not be needed for the later stages, and it only affects the results at the inner $1 / 2$ of the core region.

The relaxation parameters used are $\alpha_{0}=0.5$ for $N_{i}$, while for $D_{i}$, we use $\alpha_{1}=0.5$ for iteration $m \leq 4$ and $\alpha_{1}=0.25$ for $m>4$. The turbulence code is run for a time of $\tau_{1}=50-100 \mu$ s for each iteration, with the range of $\tau_{1}$ caused by variations in the BOUT time step for a fixed CPU time. The total time simulated over the nine BOUT runs corresponds to $700 \mu \mathrm{s}$, still much less than the transport time of $\tau_{0}=10 \mathrm{~ms}$. Each BOUT iteration takes 8 hours using 64 processors on a parallel computer utilizing $667 \mathrm{MHz}$ CPUs. The UEDGE iteration time is negligible, typically less than a few minutes on 1 CPU.

The radial density profiles obtained at the outer midplane for 9 iterations are shown in Fig. 3a. The corre-
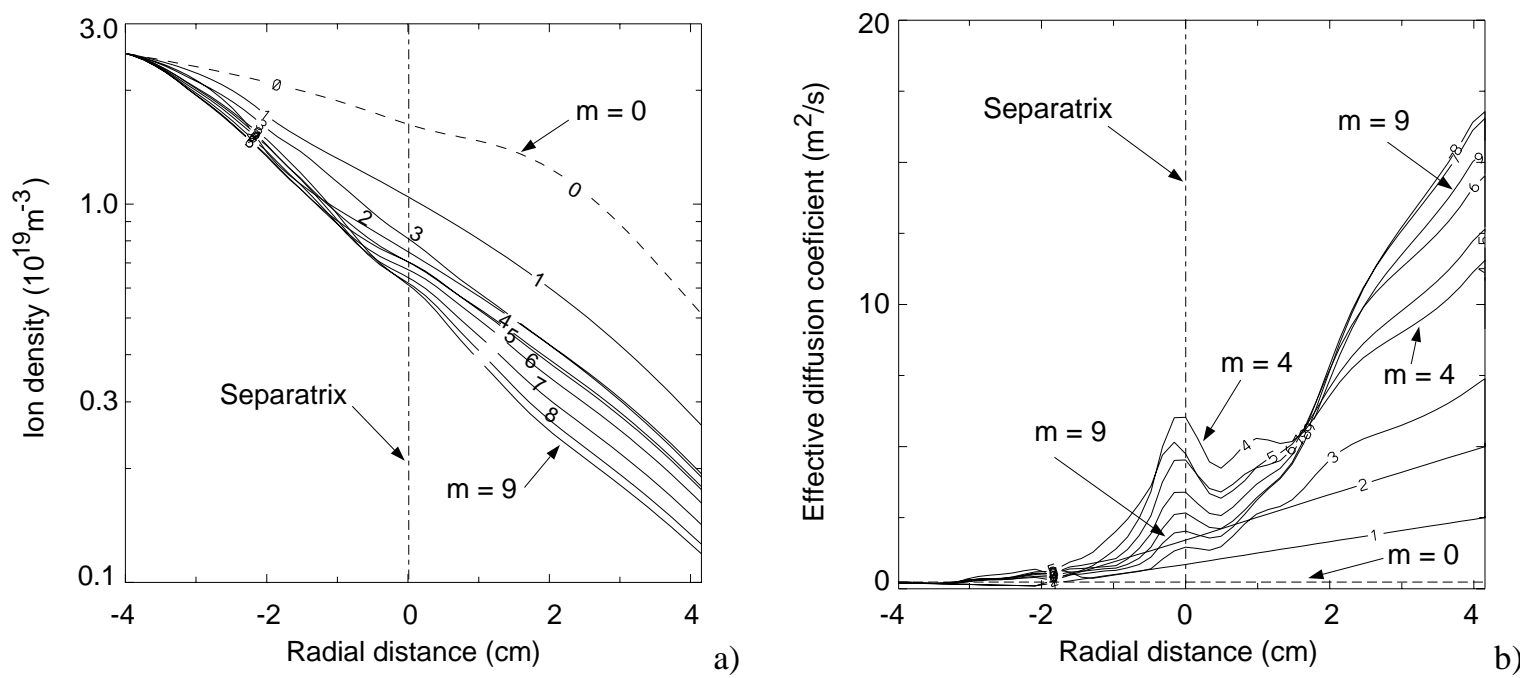

Fig. 3 Iteration history of a) midplane density, and b) effective diffusion coefficient using the algorithm from Eqs. (5-7) 
sponding diffusion coefficients are plotted in Fig. 3b. For the first two iterations $(m=1,2)$, a linear constraint was used for the $D_{i}$ profile for $r>-0.2$, but no constraint was used for $m>2$. The initial case with $m=0$ used a constant value of $D_{i}=0.2 \mathrm{~m}^{2} / \mathrm{s}$. Note that diffusion coefficient in Fig. $3 \mathrm{~b}$ shows a strong increase for $m=4$, but then relaxes successively to $m=9$. The density profiles show a weaker variation with iteration number. The interaction appears to be converging, but there are occasional events such as seen for $m=4$ that can recur.

The final $(m=9)$ density and diffusion coefficient profiles are shown in Figs. 4a,b. For these diagnostic plots, we use the poloidal coordinate along the separatrix and the radial coordinate at the outer midplane; thus, the proper proportion on the main chamber and divertor regions is maintained, but some perspective in the privateflux and X-point regions are modified. Because the private flux region can also give rise to some numerical problems, we damp modes here; future work will seek to resolve this issue.

The diffusion coefficient in Figs. 4b clearly shows the strong ballooning character of the turbulent transport over the outer midplane region where the common sign of the density and magnetic-field radial gradients favors instability. Here $D_{i}$ peaks near the upper and lower X-points, which can be understood by the magnetic shear in these regions that give rise to a peak in the perpendicular wavenumber, $k_{\perp}$, and thus the associated large $k_{\perp} \phi / B$ cross-field velocity [1]. The few isolated peaks in $D_{i}$ are the result of $d N_{i} / d r$ becoming locally small in Eq. (3), which indicates some limitation to the diffusive model as opposed to a mixture of diffusion and convection. Nevertheless, these isolated peaks don't have a significant influence on the overall solution.

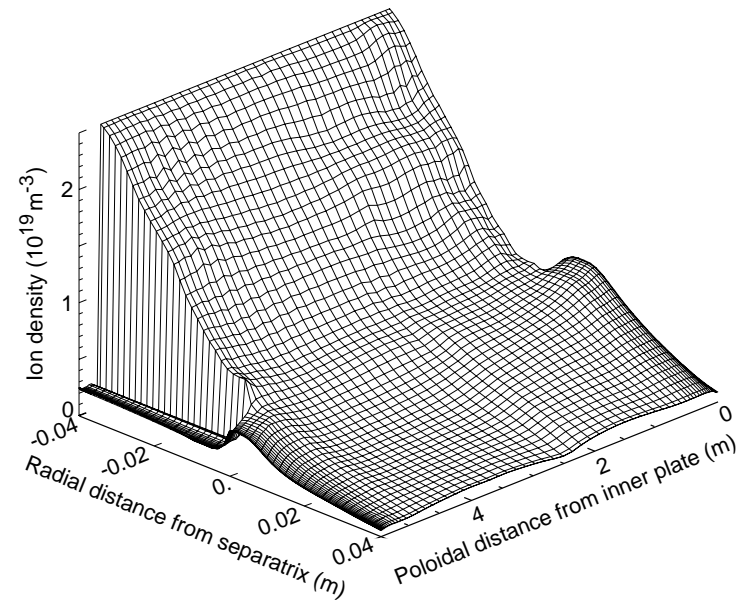

a)

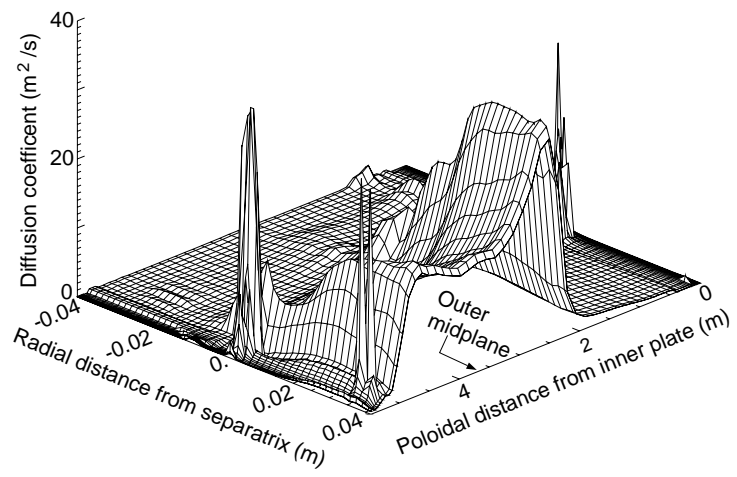

b)

Fig. 4 The final ( $m=9$ ) profiles of a), ion density, and b), effective diffusion coefficient, $D_{i}$.

Finally, we show the neutral density calculated throughout the simulation domain in Fig. 5 and the ion particle flux for $m=0$ and $m=9$ cases in Fig. 6. The neutral density is determined self-consistently, with the recycling of the ion flux to the divertor and outer walls providing the source, and the transport model described by Eqs. (12) for $k=g$ giving the spatial distribution within the plasma. The recycling sources give rise to the maxima of $N_{g}$ at the plate and outer wall boundaries. The ion flux to the outer wall in b) peaks near the upper X-point and is large between this region and the lower X-point as expected from the broad peak in $D_{i}$ shown in Fig. 4b. The integrated ion flux to the outer wall is $4.5 \mathrm{kA}$, while that to the two divertor plates is $1.7 \mathrm{kA}$.

\section{Discussion and summary}

We have presented an algorithm and shown the initial results of coupling the 2D UEDGE transport and 3D BOUT turbulence codes. For simplicity, the direct coupling has involved only the ion density profile (from UEDGE) and the associated radial particle flux (from BOUT), although UEDGE also evolves the parallel ion velocity and the neutral density, while BOUT also calculates fluctuations in parallel velocities, ion and electron temperatures, and electrostatic potential as part of the turbulence.

The results indicate that the iteration procedure approaches a quasi-steady-state, although substantial noise and only a moderate number of iterations leaves a firm conclusion beyond the reach of this paper. These questions will be addressed by continued work on this problem. What does seem clear is the strong transport in the region 


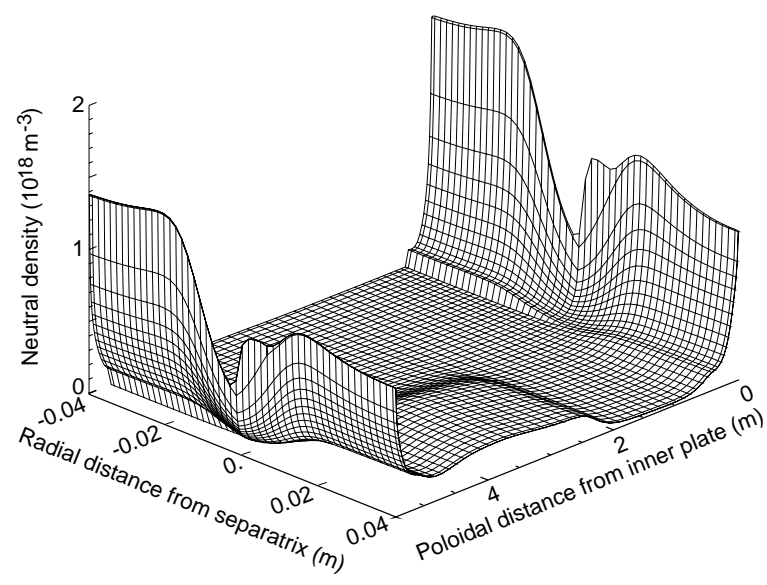

Fig. 5 Final $(m=9)$ profile neutral density, $N_{g}$.

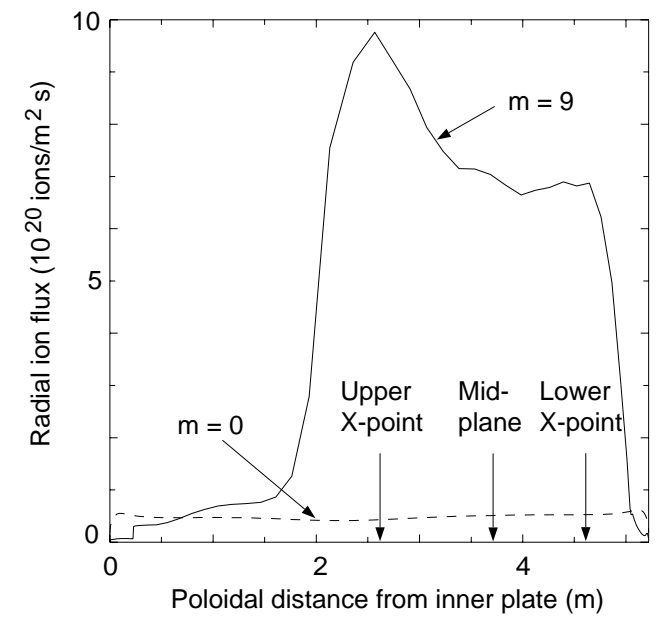

Fig. 6 Final ion particle flux along the outer wall from inner to outer divertors, with flux for constant $D_{i}=$ $0.2 \mathrm{~m}^{2} / \mathrm{s}$ at $m=0$ shown for comparison

beyond the separatrix that increases toward the wall. This is qualitatively consistent with the observations and modeling of strong transport for some tokamak discharges $[6,7,8]$. However, our coupled simulation does not provide any information on what conditions lead to this strong radial transport. On the other hand, previous BOUT simulations with ad hoc background profiles have also shown strong SOL transport [9], especially at increasing plasma resistivity (higher $N_{i}$, lower $T_{e}$ ).

Extension of the methods used here to other variables, notably $V_{\| i}, T_{e, i}$ and $\phi$, will be pursued. There should be no direct increase in computation cost since the fluxes from these quantities are already available from the present simulations, but have not yet be used in order to build an understanding of the simpler system coupled here. Inclusion of the radial electric field inside the separatrix should allow formation of H-mode transport barriers.

An additional aide in the coupling of turbulence and transport is to allow BOUT to provide its own background by evolving the toroidally-averaged quantities. The BOUT portion of this strategy is illustrated in a separate paper $[10]$ in these proceedings. In fact, both in that work, and here, we occasionally $(\sim 0.5 \mathrm{~ms})$ observed a strong localized increase in fluctuations in the core region, that then dissipate. The physical (or numerical) origin of these fluctuations is being investigated. If these enhancements are physical, BOUT can be used to give the profile response. However, for the long times ( $>5 \mathrm{~ms}$ ) associated with recycling and neutral profile adjustment, as illustrated in this paper, coupling to a transport code appears an realizable and efficient solution.

Acknowledgements We thank W.M. Nevins and D.D. Ryutov of helpful discussions. This work was performed under the auspices of the U.S. Dept. of Energy by the University of California Lawrence Livermore National Laboratory under contract No. W-7405-Eng-48 and was supported as LLNL LDRD project 03-ERD-009.

\section{References}

[1] X.Q. Xu, R.H. Cohen, T.D. Rognlien, and J.R. Myra, Phys. Plasmas 7, 1951 (2000).

[2] T.D. Rognlien, D.D. Ryutov, N. Mattor, and G.D. Porter, Phys. Plasmas 6, 1851 (1999).

[3] A.I. Shestakov, R.H. Cohen, J.A. Crotinger, L.L. LoDestro, A. Tarditi, and X.Q. Xu, J. Comp. Phys. 185, 399 (2003).

[4] S.I. Braginskii, Transport processes in a plasma, Reviews of Plasma Physics, Vol. 1, Ed. M.A. Leontovich (Consultants Bureau, New York, 1965), p. 205.

[5] R.H. Cohen, N. Mattor, and X.Q. Xu, Contrib. Plasma Phys. 34, 232 (1994).

[6] B. LaBombard, M.V. Umansky, R.L. Boivin et al., Nucl. Fusion 40, 2041 (2000).

[7] M.V. Umansky, S.I. Krasheninnikov, B. LaBombard et al., Phys. Plasmas 6, 2793 (1999).

[8] S.I. Krasheninnikov, Phys. Lett. A 283, 368 (2001).

[9] X.Q. Xu, W.M. Nevins, T.D. Rognlien et al., Phys. Plasmas 10, 1773 (2003)

[10] X.Q. Xu et al., "Correlation of density pedestal width and neutral penetration length," paper at this conference. 